\title{
Use of flexible plastic film isolators in performing potentially hazardous necropsies
}

\author{
PC TREXLER, AM GILMOUR \\ From the Robens Institute, University of Surrey, Guildford and the Department of Histopathology, District \\ General Hospital, Eastbourne
}

SUMMARY A number of necropsies were performed with the body completely isolated in a flexible film isolator in order to determine the practicability of such a method in protecting the post mortem room staff from the risk of infection from the corpse. It is considered that necropsies can be carried out using such an isolator without hindrance to either manual skill or vision and that a high degree of safety is achieved. The isolator may also be used to carry out necropsies on decomposed bodies.

The risk to post mortem room staff of infection from the corpse has long been appreciated and the current attention generated by the Howie Code of Practice on safety in mortuaries led us to investigate the possibility of eliminating such risks by isolating the body within a sealed plastic envelope. One of us (PCT) has had considerable experience of this equipment in the clinical situation where the bed isolator has been used, both to protect immunosuppressed patients from cross-infection, ${ }^{1}$ and also to isolate patients with dangerous infections. $^{2}$ Extensive microbiological tests have demonstrated the efficacy of the flexible film isolator. $^{3}$

\section{The flexible film isolator}

This is a sealed envelope of transparent flexible polyvinylchloride (PVC) film the shape being that of a truncated ridge tent $(2.3 \mathrm{~m}$ long, $0.9 \mathrm{~m}$ wide, $0.9 \mathrm{~m}$ high). The base of the envelope rests on the necropsy table and the unit is attached at its sides and supported from its roof by an adjustable frame of lightweight plastic covered steel tubes. A zip opening along the roof and ends allows entry and exit of the body. Access to the body is provided by three pairs of sleeves with replaceable gloves, one on each side of the envelope and another on the head end. The side sleeves are sealed to the envelope around overlapping openings so as to make the length of the body accessible. The right-hand sleeve at the head end has an evagination on the forearm region to receive an electric saw so arranged that only the

Accepted for publication 10 November 1982 sterilisable saw blade is actually within the envelope. Instruments and specimens are removed after necropsy into an evaginated sleeve which is then sealed. A water hose can be passed into the envelope via a flexible film cone in the envelope wall which is cut and then taped shut after the hose has been pulled in. The body and enclosing envelope rest on an inclined wooden board allowing gravity drainage. The effluent is collected in a plastic tank with a capacity of approximately $200 \mathrm{l}$ and a sealed lid to permit an air-tight connection to the envelope. The tank contains a phenolic germicide and after an appropriate holding period a tap is opened and the contents poured down the drain.

A negative pressure is maintained within the envelope by means of a small blower $(40 \mathrm{l} / \mathrm{s}$ at 25 $\mathrm{mm}$ water pressure) connected to the isolator through a pressure control mechanism and an airfilter. Sufficient air-pressure differential should be maintained to ensure that the pressure remains negative in order to prevent the escape of an aerosol in case of a puncture. ${ }^{3}$ The airfilter (LF $40^{*}$ ) sterilises the air as it leaves the envelope and is disposable. It can be decontaminated by means of a germicide-for example, hypochlorite solution used to treat the envelope or it can be withdrawn into a plastic sleeve for autoclaving.

The pathologist works with his arms inside wide invaginating sleeves wearing two pairs of rubber gloves. All parts of the body are accessible and neither manual dexterity nor vision are impaired. The body is opened, eviscerated and organs dissected by the pathologist. Reconstruction of the body is performed by the post mortem room technicians.

The instruments, after washing, are placed in the 


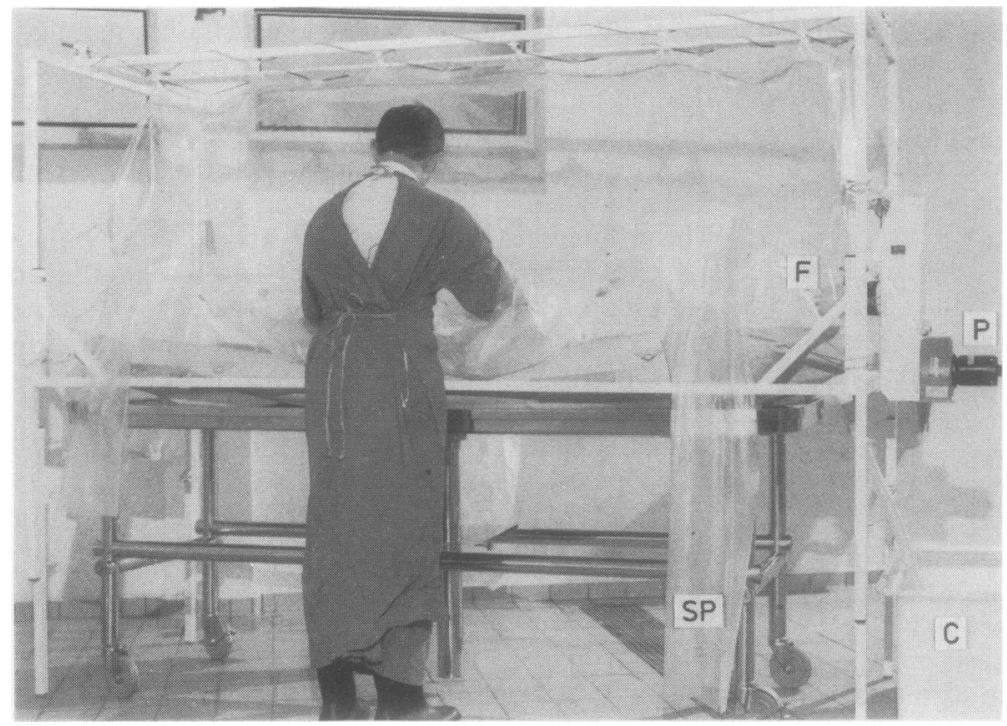

Fig. 1 The transparent plastic envelope $(E)$ is attached to the frame $(F)$ and rests on the mortuary table. The sleeves $(S)$ are shown in the evaginated position to demonstrate the length and width at the base.

Fig. 2 This shows the operator with his arms inside the sleeves. The long evaginated sleeve to his right $(S P)$ collects specimens and instruments after use. Negative pressure is maintained by pump $(P)$, the air removed first passing through filter $(F)$. All effluent fluids are collected in container $(C)$.

evaginated sleeve along with specimens for histology and microbiology in their appropriate containers. The isolator envelope and the body are then thoroughly washed using the hose to remove all traces of body fluids. The body and isolator are then sanitised by instilling formalin and allowing several hours for it to act. Alternately the envelope may be collapsed around the body by extracting air through the filter, which is then sealed off, removed, and the body with the enclosing envelope disposed of.

\section{Discussion}

We envisage the isolator being used in cases of sus- $-\frac{0}{0}$ pected tuberculosis, septicaemia and hepatitis $\mathrm{B} \stackrel{?}{?}$ virus infection which the pathologist might consider $\square$ unacceptably hazardous to staff. We feel, however, that post mortems on patients dying of a highly infectious and dangerous disease such as the acute $\overline{\mathrm{D}}$ viral haemorrhagic fevers should only be carried out in a special isolation laboratory. 
The isolator may also be used to great effect for post mortems on decomposed bodies. Protected from the unpleasant odours of such cases, the pathologist, we feel sure, will perform a more prolonged and thorough examination.

\section{Conclusions}

Our experience has shown us that post mortems can be performed in the isolator in the normal fashion with little extra difficulty or time required. The risk of airborne infection is virtually eliminated although prevention of inoculation will always depend on careful work by the pathologist and his technician.

We believe that the flexible film body. isolator can be of considerable value to pathologists and we hope that it will be commercially developed and made available to pathologists in the near future.

We wish to acknowledge the support provided by the Operations Work group, Department of Health and Social Security and Vickers Medical. Also we thank Mr BJ Hoad and Mrs L Huggett for patient assistance in the mortuary and Mr DM Manners and Mrs LA Dove for photographic and secretarial assistance.

\section{References}

1 Trexler PC, Spiers ASD, Gaya H. Plastic isolators for treatment of acute leukaemia patients under " germ-free" conditions. $\mathrm{Br}$ Med J 1975;4:549-52.

2 Trexler PC, Emand RTD, Evans B. Negative-pressure plastic isolator for patients with dangerous infections. $\mathrm{Br}$ Med J 1977;ii:559.

${ }^{3}$ Hutchinson JGP, Gray J, Hewett JH, Emand RTD, Brandon Evans, Trexler PC. The safety of the Trexler isolator as judged by some physical and biological criteria: A report of experimental work at two centres. J Hyg (Camb) 1978;81:311-9.

Requests for reprints to: Dr AM Gilmour, Consultant Histopathologist, District General Hospital, Kings Drive, Eastbourne, East Sussex BN21 2UD, England. 